\title{
Propagation des fronts de fissure plane dans les matériaux fragiles hétérogènes de dimensions finies
}

\author{
Sylvain Patinet ${ }^{1, a}$, Jö̈l Frelat ${ }^{2}$, Veronique Lazarus ${ }^{3}$ et Damien VandembroucQ ${ }^{1}$ \\ 1 Laboratoire PMMH, UMR 7636 CNRS/ESPCI/P6/P7, 10 rue Vauquelin, 75231 Paris Cedex 05, France \\ 2 UPMC Univ Paris 6, UMR 7190 (IJLRDA), Boîte courrier 161-2, 4 place Jussieu, 75005 Paris, France \\ 3 UPMC Univ Paris 6, Univ. Paris-Sud, CNRS, UMR 7608, Lab. FAST, Bât. 502, Campus Univ., 91405 Orsay, France
}

Reçu le 1 mai 2011, accepté le 20 mai 2011

\begin{abstract}
Résumé - Notre approche vise à tester les modèles classiques de piègage de fissure pour des motifs de ténacité contrôlée et des géométries d'éprouvette réalistes, c'est-à-dire de tailles finies. Nous modélisons notamment l'effet de l'épaisseur du matériau. Les prédictions du modèle théorique développé dans [L. Legrand, S. Patinet, J.-B. Leblond, J. Frelat, V. Lazarus, D. Vandembroucq, Coplanar perturbation of a crack lying on the mid-plane of a plate, Int. J. Frac.] sont validées par des calculs de type éléments-finis. Ces derniers convergent en fonction de la fréquence de perturbation du front de fissure vers deux régimes asymptotiques : milieu semi-infini et plaque mince. Dans le cas d'une fissure interagissant avec un défaut unique, nous confrontons nos calculs à une configuration expérimentale de fissuration. Nous montrons une remarquable amélioration des prédictions des modèles de ligne élastique par la prise en compte de l'épaisseur de l'éprouvette.
\end{abstract}

Mots clés : Fissure / plaque / éléments-finis / ligne élastique

\begin{abstract}
Propagation of planar crack fronts in heterogeneous brittle materials of finite dimensions. Our approach consists in testing the classical crack-pinning models for controlled toughness patterns and realistic sample geometries, i.e. of finite size. We model the effect of sample thickness on the stress intensity factor at the crack tip. The predictions of the theoretical model developed in [L. Legrand, S. Patinet, J.-B. Leblond, J. Frelat, V. Lazarus, D. Vandembroucq, Coplanar perturbation of a crack lying on the mid-plane of a plate, Int. J. Frac.] are validated by finite-element calculations. These calculations converge as a function of the crack front perturbation frequency towards two asymptotic regimes: semiinfinite medium and thin plate. In the case of a crack interacting with a single defect, we compare our calculations with an experimental crack configuration. We show a remarkable improvement of elastic line model predictions when the sample thickness is taken into account.
\end{abstract}

Key words: Crack / plate / finite-elements / elastic line

\section{Introduction}

La prédiction du seuil de propagation des fissures est une problématique essentielle de la science des matériaux. Elle détermine la qualité de la conception des structures ainsi que leur durabilité. Depuis le début des années 90, des modèles de transition critique basés sur la physique statistique ont été abondamment utilisés pour décrire la fracture des matériaux hétérogènes [2]. L'avancée des fissures est modélisée par la dynamique d'une ligne élastique dans un milieu infini interagissant avec un potentiel aléatoire correspondant au contraste de ténacité des

\footnotetext{
a Auteur pour correspondance :

sylvain.patinet@gmail.com
}

matériaux. Ce contraste est issu des hétérogénéités chimiques ou structurelles du matériau lesquelles induisent une variation locale de la ténacité se traduisant par un ancrage des fissures dans les zones les plus tenaces. Motivée par la promesse de décrire un comportement universel, l'étude de la propagation des fissures s'est essentiellement concentrée sur l'invariance des lois d'échelles en décrivant la dynamique des fissures au voisinage du seuil de propagation $[3,4]$. Cette focalisation s'est faite en général au détriment de l'étude du seuil de propagation qui, au contraire, dépend fortement des détails du système. La ténacité macroscopique d'un matériau est en effet fonction du type de désordre, du mode de rupture ainsi que des dimensions du système [5]. C'est ce qui justifie nos travaux 
(a)

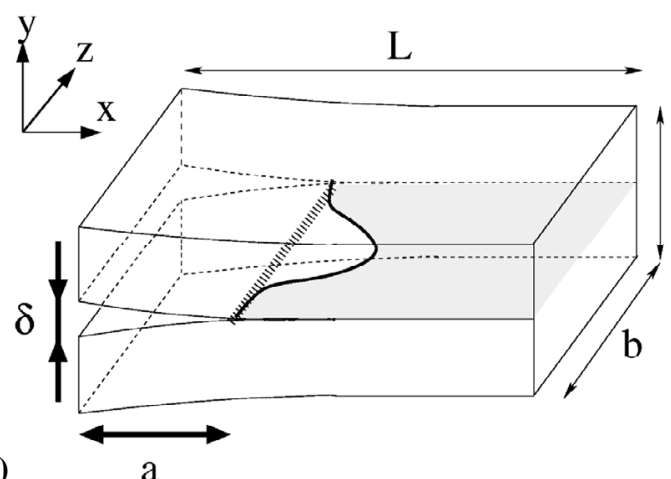

$2 \mathrm{~h}$

(b)

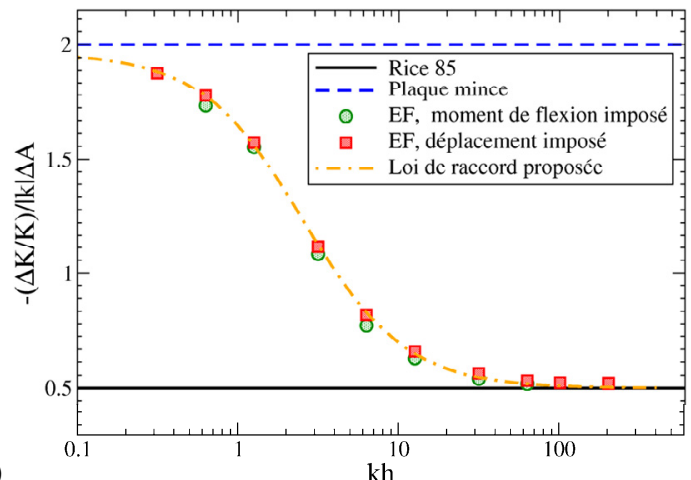

Fig. 1. (a) Schéma d'un essai de clivage représentant une fissure planaire dans une éprouvette de taille finie d'épaisseur $h$. (b) Amplitude normalisée du taux de restitution d'énergie pour une perturbation sinusoïdale de la position du front de fissure en fonction de la fréquence de la perturbation, $k=2 \pi / \lambda$, normalisée par rapport à l'épaisseur de l'éprouvette. Les courbes et les symboles correspondent respectivement aux différentes approches théoriques et aux calculs numériques par éléments-finis tels que reportés dans [1].

dont la motivation est de se rapprocher de la prédiction la plus quantitative possible du seuil de propagation des fissures. Notre approche vise à tester les modèles classiques d'accrochage de fissure pour des motifs de ténacité contrôlée et des géométries d'éprouvette réalistes, c'est-à-dire de tailles finies. Pour cela, nous confrontons les prédictions théoriques (mécanique élastique linéaire de la rupture, lignes élastiques) aux calculs numériques (éléments-finis) ainsi qu'aux expériences dans le cadre d'un essai classique de clivage schématisé sur la figure 1a.

Dans un premier temps, nous modélisons l'effet de l'épaisseur ( $h$ sur la Fig. 1a) du matériau sur le facteur d'intensité des contraintes en pointe de fissure $K(z)$. Cette étude théorique est réalisée en collaboration avec Leblond et Legrand (Institut Jean Le Rond d'Alembert/UPMC) [1]. Les calculs théoriques de la variation du facteur d'intensité des contraintes $\delta K(z)$ en réponse à une perturbation du front de fissure $\delta a(z)$ pour différentes longueurs d'onde $\lambda$ montrent l'existence de deux régimes asymptotiques : milieu semi-infini et géométrie plaque $[1,6]$. Cette prédiction est validée par des calculs de type éléments-finis (EF) qui convergent vers les deux régimes asymptotiques en fonction de la fréquence de la perturbation (Fig. 1b). À partir des calculs par EF nous proposons une loi de raccordement dans le régime intermédiaire pour des éprouvettes d'épaisseur arbitraire.

Les données issues d'un montage de type clivage développé par Alzate et Dalmas (Surface du Verre et Interfaces - unité mixte CNRS/Saint-Gobain) [7] nous permettent de réaliser une première comparaison entre expérience et modèle de piégeage. Dans le cas d'une fissure interagissant avec un défaut unique (Fig. 2), nous calculons par la méthode des EF les facteurs d'intensité des contraintes locaux correspondant à la morphologie $\mathrm{du}$ front de fissure expérimental. Nous montrons qu'il est nécessaire de prendre en compte l'épaisseur des éprouvettes dans l'optique d'une prédiction quantitative du contraste de ténacité par les modèles de ligne élastique (Fig. 3).

\section{Noyaux élastiques d'une fissure dans une plaque d'épaisseur finie}

\subsection{Approches théoriques : milieu semi-infini et plaque mince}

Nous résumons ici les propriétés des noyaux élastiques décrivant les fissures pour des systèmes de dimensions différentes par rapport à la longueur d'onde $\lambda$ de perturbation du front de fissure. Un lecteur intéressé par les dérivations au premier ordre en perturbation de ces formules pourra se reporter aux références $[1,6,8]$.

\section{Solution de Rice pour un corps infini 3D}

Rice a calculé l'expression théorique de la variation du facteur d'intensité des contraintes $\delta K(z)$ le long d'une fissure plane en fonction d'une perturbation $\delta a(z)$ de la position du front de fissure. Il a dérivé cette expression pour une fissure semi-infinie dans un corps infini [8]. Du point de vue des essais classiques de clivage (Fig. 1a), une telle schématisation géométrique est acceptable si la distance caractéristique de variation $\lambda$ de la perturbation du front de fissure remplit les conditions : $\lambda \ll h$ et $\lambda \ll a$. La première de ces conditions implique que la plaque doit être très épaisse. Pourvu que ces hypothèses soient satisfaites et pour un chargement invariant le long du front dans la direction $z, \widehat{\delta K}(k)$ est donné dans l'espace de Fourier par :

$$
\begin{aligned}
\frac{\widehat{\delta K}(k)}{K_{0}}= & F_{\text {Rice }}(k, a) \widehat{\delta a}(k) \\
& \text { avec } \quad F_{\text {Rice }}(k, a)=\left(-\frac{|k|}{2}+\frac{1}{K_{0}} \frac{\mathrm{d} K}{\mathrm{~d} a}\right)
\end{aligned}
$$

où $2 \pi /|k|=\lambda$ correspond à la longueur d'onde de la perturbation du front ( $k$ correspondant à la fréquence de la perturbation) et $K_{0}$ au facteur d'intensité des contraintes d'un front droit de position moyenne $a$. 

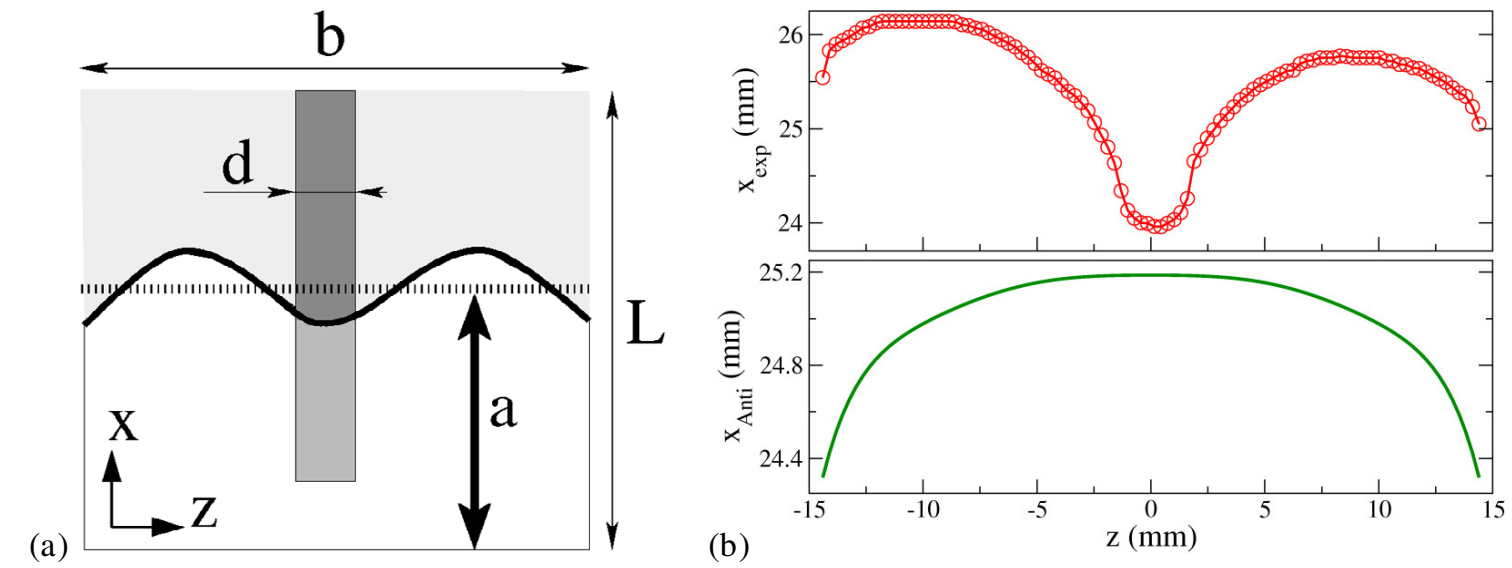

Fig. 2. (a) Schéma d'une fissure d'interface interagissant avec un défaut de taille contrôlée. (b) Dessus : position expérimentale d'une fissure interagissant avec un défaut. Dessous : position d'une fissure obtenue par éléments-finis dans un milieu homogène équivalent dont la courbure provient du couplage anticlastique.

\section{Solution pour une plaque mince}

Nous avons développé la méthode de Rice en l'adaptant au cas d'une fissure planaire semi-infinie située dans le plan médian d'une plaque très mince [1]. Nous considérons donc la limite répondant aux conditions : $h \ll \lambda$ et $h \ll a$. Suivant ces hypothèses, la perturbation de la fissure peut être traitée dans le cadre de la théorie des plaques minces de Love-Kirchhoff ce qui réduit la complexité du problème. La variation du facteur d'intensité des contraintes dans l'espace de Fourier s'écrit alors :

$$
\begin{aligned}
& \frac{\widehat{\delta K}(k)}{K_{0}}=F_{\text {plaque }}(k a) \widehat{\delta a}(k) \\
& \text { avec } \quad F_{\text {plaque }}(k a)=\frac{1}{a}\left(\frac{2 k a \cosh (2 k a)-\sinh (2 k a)}{2 k a-\sinh (2 k a)}\right)
\end{aligned}
$$

\section{2 Étude numérique d'une plaque d'épaisseur arbitraire}

Nous allons maintenant envisager le cas général d'une plaque d'épaisseur arbitraire. Par conséquent, ni les hypothèses géométriques du milieu semi-infini (1) ni celles de la théorie des plaques (2) ne seront imposées. Notre objectif est double : montrer la transition entre les deux régimes décrits précédemment et proposer une loi les raccordant.

Nous introduisons toutefois l'hypothèse selon laquelle : $h \ll a$. Cette condition est en effet souvent remplie dans la pratique et des simplifications considérables en résultent. Notons immédiatement, par exemple, qu'avec une telle hypothèse, $\lambda \ll h$ suffit pour justifier l'applicabilité de la formule de Rice (1), tandis que $\lambda \gg h$ suffit pour justifier l'applicabilité de la formule (2). Nous cherchons à construire une formule d'interpolation dans l'espace de Fourier entre les expressions (1) et (2) sous la forme :

$$
\begin{aligned}
\frac{\widehat{\delta K}(k)}{K_{0}}=[(1- & \left.\theta(k h)) F_{\text {Rice }}(k, a)+\theta(k h) F_{\text {plaque }}(k a)\right] \\
& \times \widehat{\delta a}(k)=F_{\text {raccord }}(k, a, k h) \widehat{\delta a}(k)
\end{aligned}
$$

où $\theta(k h)$ désigne une fonction du nombre d'onde réduit $q=k h$ à déterminer, obéissant aux propriétés :

$$
\begin{aligned}
& \theta(k h) \rightarrow 1 \quad \text { pour } \quad k h \rightarrow 0 \\
& \text { et } \quad \theta(k h) \rightarrow 0 \quad \text { pour } \quad k h \rightarrow \infty
\end{aligned}
$$

La formule (3) possède les caractéristiques souhaitées. En effet si $\lambda \ll h$ tel que $\theta(k h) \rightarrow 0$ l'expression (3) se réduit à (1). Inversement, si $\lambda \ll h$ tel que $\theta(k h) \rightarrow \infty$, l'expression (3) se réduit à (2).

La fonction $\theta(k h)$ est déterminée en effectuant des calculs par EF élastiques de plaque fissurée de dimension finie. La longueur de la fissure $a$ et la longueur du ligament non fissuré $L-a$ sont choisies beaucoup plus grandes que l'épaisseur $h$ de la plaque. Deux configurations du front sont considérées, une droite et une autre avec une légère ondulation sinusoïdale dans le plan de longueur d'onde $\lambda$. En raison de la périodicité du problème dans la direction $z$, seule une tranche de matière se trouvant entre les plans $z=0$ et $z=\lambda$ est maillée, des conditions aux limites périodiques étant imposées sur ces plans $(\lambda=b$ sur Fig. 1a). Les oscillations du facteur d'intensité de contraintes locales le long du front de fissure $\delta K(z)$ sont déterminées en comparant les valeurs prises par ce facteur dans les configurations originales et perturbées.

Un test de pertinence de l'approche perturbative au premier ordre réside dans le fait que les oscillations de $\delta K(z)$ gardent une forme sinusoïdale pour une avancée locale de la fissure $\delta a(z)$ elle aussi sinusoïdale. Ce test sert à fixer une valeur maximale admissible de l'amplitude de variation $\delta a(z)_{\max }=\Delta A$. La valeur du ratio de $\delta K(z) / \delta a(z)$ est déduite de celle du rapport des amplitudes de variation $\Delta K / \Delta A$. Plusieurs valeurs de $\lambda$ sont considérées, chacune d'entre elles comparable à $h$ et beaucoup plus petite que $a$. La fonction $\theta(k h)$ peut donc être liée à la quantité $X=\left(\Delta K / K_{0}\right) /|k| \Delta A$. Notons en particulier que du fait des équations (1) et (2), $X$ doit prendre les valeurs 2 et $1 / 2$ dans les limites $|k h| \ll 1$ et $|k h| \gg 1$ respectivement.

Les calculs sont effectués avec le code d'EF CAST3M développé au Commissariat à l'Énergie Atomique. Deux 

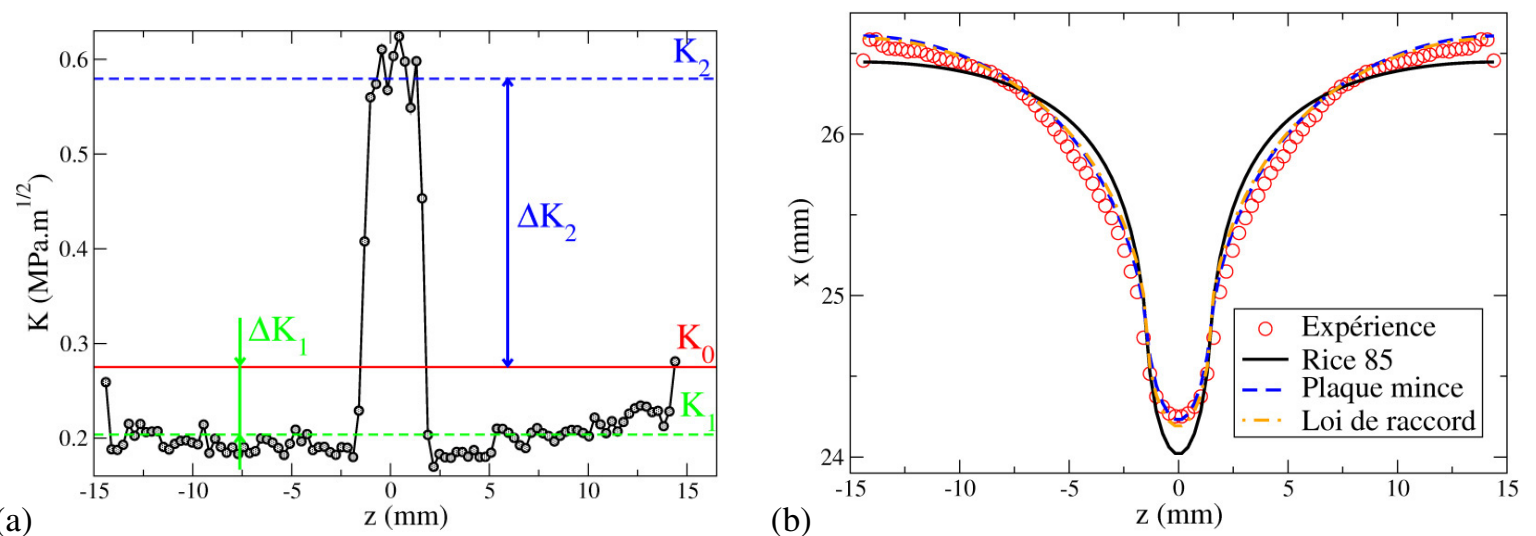

Fig. 3. (a) Taux de restitution d'énergie calculé par la méthode des éléments-finis en fonction de la position longitudinale le long du front de fissure. (b) Position de la fissure. Les données expérimentales corrigées (cercles) sont comparées aux différents modèles de ligne élastique (courbes continues et discontinues).

types de calculs sont effectués avec différentes conditions aux limites sur les moitiés supérieure et inférieure de la limite gauche de la plaque fissurée : déplacements verticaux imposés et moments de flexion imposés. Les conditions aux limites du premier type correspondent exactement à celles utilisées dans les expériences réelles. Les deux types de conditions sont censés donner des résultats identiques pour des plaques suffisamment élancées. C'est ce qui sert de test pour savoir si les dimensions considérées sont suffisantes. Les deux types de calculs utilisent également différentes dimensions, maillages et types d'éléments bien que l'épaisseur des plaques soit égale à $1 \mathrm{~mm}$ dans les deux cas. En condition de déplacements imposés, la distance a entre le côté gauche et le front non perturbé est de $100 \mathrm{~mm}$ ou $200 \mathrm{~mm}$, selon que $\lambda$ est inférieure ou supérieure à $10 \mathrm{~mm}$. Le maillage est composé de 449760 ou 646440 éléments cubiques bilinéaires à 8-nœuds En condition de moments imposés, qui exigent des maillages plus petits, la distance $a$ est de $50 \mathrm{~mm}$ et le maillage est constitué de 182400 éléments prismatiques bilinéaires à 6-nœuds. Dans les deux cas, les éléments sont strictement cubiques ou prismatiques pour les configurations rectilignes $\mathrm{du}$ front. Pour les configurations perturbées le même maillage est utilisé avec une légère distorsion des éléments. La valeur du coefficient de Poisson est la même dans tous les cas, soit $\nu=0,3$. Les valeurs locales du facteur d'intensité des contraintes le long du front de fissure sont évaluées à l'aide de la méthode « G-theta » développée par Destuynder et al. [9] dont la précision est bien établie.

La figure $1 \mathrm{~b}$ montre les valeurs numériques de la quantité $X$ en fonction de $|k h|$ Les deux types de conditions aux limites donnent des résultats identiques ce qui montre que les dimensions des plaques considérées sont assez grandes. Comme prévu, la quantité $X$ tend vers des valeurs égales à 2 (solution plaque mince) et $1 / 2$ (solution $3 \mathrm{D}$ ) dans les limites $k h \rightarrow 0$ et $k h \rightarrow \infty$ respectivement. Nous observons qu'une augmentation de $\lambda$ à partir de valeurs beaucoup plus petites que $h$ à des valeurs beaucoup plus grandes que l'épaisseur induit une augmentation de $X$. Ce résultat contredit complètement l'idée intuitive que l'épaisseur de la plaque devrait représenter une longueur de coupure au-dessus de laquelle le rapport $\delta K(z) / \delta a(z)$ diminue rapidement. Le rôle de la longueur de coupure est donc joué uniquement par la distance $a$ dans ce problème. Il est également montré sur la figure $1 \mathrm{~b}$ que les résultats numériques peuvent être représentés avec précision par la formule simple de raccord $\theta(k h) \simeq 1 /\left(1+1 / 3|k h|^{(4 / 3)}\right)$ qui est donc adoptée ci-après.

\section{Application à un essai de clivage}

\subsection{Conditions expérimentales}

L'utilisation d'un montage de type clivage développé au laboratoire Saint-Gobain Recherche permet de mesurer l'adhésion de couches minces sur les verres et de suivre la propagation de fissures purement interfaciales [7].

Grâce à l'utilisation d'un masque lors du dépôt d'une couche métallique, une bande de défaut unique, dans laquelle on trouve une interface forte, est introduite de façon contrôlée. Le système étudié est schématisé sur la figure 2a. L'éprouvette présente une adhésion interfaciale hétérogène avec une valeur faible pour une interface verre/Ag (gris clair) et une valeur forte pour une interface verre/Si3N4 (gris foncé).

L'instrumentation optique et mécanique fournit un moyen de quantifier a posteriori le facteur d'intensité des contraintes et la ténacité interfaciale. La propagation de la fissure est pilotée par l'introduction d'une lame qui sépare les plaques de part et d'autre de l'interface à fissurer. Cette configuration expérimentale revient à imposer un déplacement sur les bords de l'échantillon. Nous adopterons donc par la suite des conditions aux limites en déplacements imposés pour les calculs numériques d'EF. La propagation du front de fissure est réalisée en régime quasi-statique, c'est-à-dire que chaque incrémentation de l'ouverture n'est appliquée qu'à la suite de l'arrêt complet $\mathrm{du}$ front de fissure. La position du front est ainsi mesurée pour une ouverture donnée. Positions du front et 
Tableau 1. Contrastes de ténacité.

\begin{tabular}{ccccc}
\hline Méthode de calcul & Éléments-finis & $\begin{array}{c}\text { Rice 85 } \\
\text { Équation (1) }\end{array}$ & $\begin{array}{c}\text { Plaque mince } \\
\text { Équation }(2)\end{array}$ & $\begin{array}{c}\text { Loi de raccord } \\
\text { Équation (3) }\end{array}$ \\
\hline$\Delta K_{1} / K_{0}$ & $-0,22 \pm 0,03$ & $-0,07$ & $-0,17$ & $-0,17$ \\
\hline$\Delta K_{2} / K_{0}$ & $1,67 \pm 0,2$ & 0,63 & 1,52 & 1,48 \\
\hline
\end{tabular}

ouvertures constituent, avec les dimensions géométriques de l'éprouvette et les constantes d'élasticité du verre, les paramètres physiques du problème utilisés dans les calculs théoriques.

Un front de fissure dans un milieu homogène se propage théoriquement en conservant une forme droite. Ici, la modification de la morphologie du front est le résultat de l'augmentation locale de la ténacité dans la zone de renfort. Comme reporté sur la figure 2, on observe que la fissure est piégée par le défaut. Ce système expérimental de piégeage d'une fissure au moyen d'une zone de ténacité élevée pour une géométrie connue nous permet de réaliser une comparaison avec les modèles de ligne élastique décrits dans la section 1.

\subsection{Comparaison avec les modèles de ligne élastique}

Avant de mettre en œuvre les modèles de ligne élastique, deux types de calculs par EF sont réalisés. Les premiers concernent la modélisation de la courbure du front due à l'effet anticlastique. Les seconds nous permettent d'obtenir une solution de référence de la ténacité de l'interface le long du front $K_{c}(z)$.

Les modèles de ligne élastique présentés dans la section 1 ne prennent pas en compte l'effet du couplage anticlastique dû aux bords libres sur les côtés des éprouvettes dans la direction $z$. L'effet anticlastique provient du fait que la flexion d'une plaque induit une courbure opposée perpendiculairement à la flexion principale. La plaque en flexion prend alors une forme en « selle de cheval . Au centre de l'éprouvette et en pointe de fissure, cette flexion secondaire est contrainte contrairement aux bords libres de l'éprouvette. L'état de contrainte dans la largeur de l'éprouvette varie par conséquent en fonction de la position $z$. Ce dernier passe d'un état de contraintes planes sur les bords à état mixte au centre de l'éprouvette pouvant aller jusqu'à un état de déformations planes en fonction du ratio $a / b$.

Ainsi, même pour une interface homogène sans défaut, une fissure adopte une forme courbée. Si cette échelle de longueur n'est pas prise en compte dans les modèles elle peut néanmoins être traitée par des calculs par EF. Nous calculons donc par cette méthode ce que serait la forme d'une fissure dans un milieu homogène équivalent tel que $K(z)=\left\langle K_{c}(z)\right\rangle=K_{0}$ correspondant au chargement expérimental. La courbure caractéristique obtenue est reportée sur la figure 3b. La soustraction de cette courbure nous permet de corriger les données expérimentales en éliminant l'effet anticlastique.

Dans un deuxième temps, nous calculons par la méthode des EF le facteur d'intensité des contraintes
$K(z)$ le long du front de fissure dont la position est donnée par l'expérience. Du fait de la propagation quasistatique de la fissure nous pouvons déduire qu'en régime de piégeage faible $K(z)=K_{c}(z)$ en tout point du front. Le paysage de ténacité ainsi obtenu est reporté sur la figure $3 \mathrm{a}$. On y distingue clairement la zone homogène de ténacité faible $K_{1}$ ainsi que la zone de défaut centrale caractérisée par une ténacité élevée $K_{2}$. Ce calcul est réalisé sans paramètres ajustables, en trois dimensions et dans le cadre de la mécanique élastique linéaire. Il constitue par conséquent le calcul de référence du paysage de ténacité $K_{c}(z)$ avec lequel nous comparons les prédictions des modèles de ligne élastique.

L'analyse de la morphologie d'un front de fissure requiert la description de la ténacité rencontrée le long du front de fissure $[7,10]$. En présence d'un défaut de largeur $d$, le paysage de ténacité est naturellement modélisé par :

$$
\begin{aligned}
\frac{\delta K_{c}(z)}{K_{0}}= & 1+\frac{\Delta K_{1}}{K_{0}} \Pi_{1}(x)+\frac{\Delta K_{2}}{K_{0}} \Pi_{2}(x) \\
& \text { avec } \quad\left\{\begin{array}{lll}
\Pi_{1}=0, \Pi_{2}=1 & \text { si } & |x|<d / 2 \\
\Pi_{1}=1, \Pi_{2}=0 & \text { si } & |x|>d / 2
\end{array}\right.
\end{aligned}
$$

où les $\Delta K_{i}=K_{i}-K_{0}$ représentent les contrastes de ténacité par rapport à la moyenne $K_{0}$. Sous l'hypothèse de piégeage faible $K(z)=K_{c}(z)$ en tout point du front. Nous pouvons donc écrire dans l'espace de Fourier l'égalité entre la formule (5) et les formules (1), (2) ou (3) pour en déduire la forme du front $\delta a(z)$ tel que :

$$
\begin{array}{r}
\widehat{\delta a}(k)=\frac{1}{F(k, a, k h)} \frac{\Delta K_{2}}{K_{0}}\left[-\frac{d}{b-d} \widehat{\Pi_{1}}(k)+\widehat{\Pi_{2}}(k)\right] \\
\text { avec } \quad K_{0}=\frac{K_{1}(b-d)+K_{2} d}{b}
\end{array}
$$

où $F(k, a, k h)$ correspond aux différents noyaux élastiques présentés précédemment dans la section 2. Le contraste de ténacité est ajusté à partir de la formule (6) de façon à reproduire la forme du front de fissure expérimentale corrigée de l'effet anticlastique. Les morphologies des fronts et les contrastes de ténacité obtenus à partir des différents noyaux élastiques sont reportés sur la figure $3 \mathrm{~b}$ et dans le tableau 1 respectivement.

Nous observons une nette amélioration de la description à la fois de la forme du front et du contraste de ténacité dans le cas où l'épaisseur finie de l'éprouvette est considérée. L'erreur commise sur $\Delta K_{2} / K_{0}$ par rapport au calcul par EF passe ainsi de $62 \%$ pour le modèle de Rice (milieu semi-infini) à environ $10 \%$ pour les modèles prenant en compte l'épaisseur de la plaque. La loi de raccord 
proposée dans la section 1 n'est pas pertinente dans ce cas par rapport à la théorie des plaques. La largeur $d$ du défaut de ténacité est en effet nettement plus grande que l'épaisseur de la plaque $h$ ce qui implique des longueurs d'onde bien plus grandes également.

\section{Conclusion}

Ces travaux améliorent la description théorique de la propagation des fronts de fissure plane dans les matériaux fragiles hétérogènes de dimensions finies. Nous avons notamment mis en évidence trois résultats principaux concernant ce phénomène physique :

- l'identification numérique des deux régimes asymptotiques, milieu infini et plaque mince, en fonction de la fréquence de la perturbation du front de fissure;

- une loi de raccord permettant de décrire un front de fissure comme une ligne élastique pour une épaisseur d'éprouvette arbitraire;

- une amélioration remarquable de la prédiction du contraste de ténacité obtenu expérimentalement par un modèle de ligne élastique prenant en compte l'épaisseur de l'éprouvette.

À la condition de pouvoir décrire les ordres supérieurs de la perturbation du front de fissure [11], la démarche proposée dans ce travail permet d'envisager à terme une description précise du seuil de propagation des fissures dans les matériaux fragiles hétérogènes.

Remerciements. Les auteurs remercient vivement Lina Alzate et Davy Dalmas pour leur autorisation à utiliser une configuration expérimentale de fissure issue de leurs travaux menés au laboratoire Surface du Verre et Interfaces - unité mixte CNRS/Saint-Gobain.

\section{Références}

[1] L. Legrand, S. Patinet, J.-B. Leblond, J. Frelat, V. Lazarus, D. Vandembroucq, Coplanar perturbation of a crack lying on the mid-plane of a plate, Int. J. Frac., 2011, DOI : $10.1007 /$ s10704-011-9603-0

[2] V. Lazarus, Perturbation approaches of a planar crack in linear fracture mechanics: a review., J. Mech. Phys. Solids 59 (2011) 121-144

[3] D. Bonamy, S. Santucci, L. Ponson, Crackling dynamics in material failure as the signature of a self-organized dynamic phase transition, Phys. Rev. Lett. 101 (2008) 045501

[4] L. Laurson, S. Santucci, S. Zapperi, Avalanches and clusters in planar crack front propagation, Phys. Rev. E 81 (2010) 046116

[5] S. Roux, D. Vandembroucq, F. Hild, Effective toughness of heterogeneous brittle materials, Euro. J. Mech. A/Solids 22 (2003) 743-749

[6] L. Legrand, J.-B. Leblond, Perturbation coplanaire d'une fissure située dans le plan médian d'une plaque mince, communication $20^{\mathrm{e}}$ congrès CFM, 2011

[7] D. Dalmas, E. Barthel, D. Vandembroucq, Crack front pinning by design in planar heterogeneous interfaces, J. Mech. Phys. Solids 57 (2009) 446-457

[8] J.R. Rice, First-order variation in elastic fields due to variation in location of a planar crack front, J. Appl. Mech. 52 (1985) 571-579

[9] Ph. Destuynder, M. Djaoua, S. Lescure, Quelques remarques sur la mécanique de la rupture élastique, J. Méc. Théor. Appl. 2 (1983) 113-135

[10] J. Chopin, Statique et dynamique d'un front de fissure en milieu hétérogène, Thèse de doctorat, UPMC, 2010

[11] M. Adda-Bedia, E. Katzav, D. Vandembroucq, Secondorder variation in elastic fields of a tensile planar crack with a curved front, Phys. Rev. E 73 (2006) 035106 